\title{
The role of individual resources, health behaviour and age perception as determinants of sports participation in older age
}

\author{
Eric $\mathrm{Fa}^{\star}$ (iD) and Torsten Schlesinger \\ Faculty of Sport Science, Ruhr University Bochum, Bochum, Germany \\ ${ }^{*}$ Corresponding author. Email: eric.fass@ruhr-uni-bochum.de
}

(Accepted 29 August 2019; first published online 9 October 2019)

\begin{abstract}
Sports participation contributes to maintaining health and wellbeing in old age, hence a deeper understanding of its various determinants is necessary. Previous research has primarily focused on either the effects of individual resources or age-specific attitudes to sports participation. However, a deeper understanding of the inter-relationships between these variables is required to develop effective policies to promote sports participation in ageing societies. To address the hypothesised inter-relationships, we consider both individual resources as well as age-specific attitudes and behaviours in order to integrate them simultaneously in our analysis. Furthermore, the analysis will be differentiated according to the three social status groups. The sample contains 1,560 retired persons, aged 65 years and older, based on the fifth wave (2014) of the German Ageing Survey. Multiple Poisson regression models were estimated to test our hypotheses. After adjusting for demographic variables, greater individual resources are associated with more regular sports participation. The findings also reveal that positive age perception and healthy behaviours are related to sports participation. Slight mediation effects between the different variables can be observed. Furthermore, the effect structures vary across different social status groups. The findings show that both individual resources and age-specific behaviours and attitudes are independent determinants of sports participation in older age. Our results confirm slight inter-relationships between socio-economic resources and age-specific attitudes.
\end{abstract}

Keywords: sports participation; older age; mediation analysis; German Ageing Survey

\section{Introduction}

Given that most Western countries are experiencing increases in the proportions of older people as share of total population (demographic ageing), ${ }^{1}$ alongside the physical and health issues that accompany ageing, the maintenance of health, wellbeing and autonomy in older age is of considerable socio-political relevance. Furthermore, physical inactivity represents a global public health problem, which

(C) Cambridge University Press 2019. This is an Open Access article, distributed under the terms of the Creative Commons Attribution-NonCommercial-NoDerivatives licence (http://creativecommons.org/licenses/by-nc-nd/4.0/), which permits non-commercial re-use, distribution, and reproduction in any medium, provided the original work is unaltered and is properly cited. The written permission of Cambridge University Press must be obtained for commercial re-use or in order to create a derivative work. 
can also lead to economic burdens in ageing societies (World Health Organization (WHO), 2010; Ding et al., 2016). Accordingly, alongside other kinds of physical activities, regular participation in sport seems to be a particularly important factor for maintaining health over the lifecourse (Daskalopoulou et al., 2017). Especially in older age, sports participation can be beneficial in maintaining (self-rated) health, physical functioning and mobility, thereby contributing to autonomous and healthy ageing and a high quality of life (Conde-Sala et al., 2017; Gayman et al., 2017; Marques et al., 2018). The positive effects of regular sports activities can be shown in different health-related dimensions, such as a reduced risk of mortality (Wanner et al., 2014; Willey et al., 2015) and cardiovascular diseases (Cheng et al., 2013; Emerson and Gay, 2017), and delayed decline in cognitive functioning (Wollesen and Voelcker-Rehage, 2013; Loprinzi et al., 2018) and (mental) wellbeing (Molinari et al., 2015; Wicker and Frick, 2015; Jeckel and Sudeck, 2018).

Even though older people can benefit from sports participation, the number of older people in Germany who meet the guidelines for physical activity recommended by the WHO is insufficient (Robert Koch-Institut, 2015). According to their share of the total population, older people are under-represented in terms of sports activity (Eurostat, 2018) and so this population group does not benefit to its proportionate extent from the health-related effects of sports activities. Social inequalities are also significant, as older people with lower social status are particularly exposed to health-specific risks. In order to prevent such risks, diverse preventive actions and initiatives are developed by health policy to promote health through encouraging older people to participate in daily sports or physical activities. Such preventive concepts or programmes should seek to provide the conditions that enhance older people's opportunities to participate in sport, which in turn may increase the proportion of active older people in ageing societies. Effective-designed incentive schemes require a clear understanding of these factors and the ways in which they can determine the sports participation of older adults.

In previous research on sports participation, the effects of socio-economic factors (which are more objective measurements) and subjective measurements (such as age perception or age pictures) are often analysed separately (see the systematic review by Jenkin et al., 2017). Several studies have indicated that people with superior educational attainment and/or higher incomes are more likely to participate in sport (Breuer et al., 2010). Furthermore, this social gradient in sport and health behaviour can also be found regarding health. Given that the impacts of these different perspectives are varied, it can be assumed that explanations based solely on the unequal distribution of individual resources are limited in their reach. Not only the existence of objectively measurable resources but different ways of using and transforming these resources may prove decisive for the sports participation of older people. Yet, given that a simultaneous analysis of individual resources, health behaviour and age-specific attitudes is empirically currently absent, the underlying relationships regarding older people's sports participation remain unclear. Therefore, variability in living situations (daily life) and health- and age-related orientation (along with corresponding behavioural patterns) are not adequately addressed, even though they might significantly determine the everyday physical activities of the older adults. Moreover, the question of the status-specific determination of sports-related everyday practices is of considerable health and socio-political relevance. Therefore, 
it is important to analyse these mechanisms in relation to the sports participation of older people in a more differentiated manner.

To bridge these gaps, this paper addresses the objective and subjective determinants of sports participation in older age and attempts to consider and combine them simultaneously, based on an integrative model. This enables the potential inter-relationships between sports participation, individual resources and characteristics, and age-specific behaviours and attitudes to be observed. Therefore, the following research questions were derived:

(1) Is the effect of individual resources on sports participation in older age mediated by health behaviour and age-specific attitudes?

(2) Do the effect structures vary between different social status groups?

\section{Theoretical framework}

In order to tackle the research questions, a model was developed to account for and bring together the individual mechanisms that affect sports activities in older age: (a) differences between the resources available to individuals; and (b) age-specific health behaviours and attitudes as mechanisms of mediation.

According to newer theories of social inequality (see Bourdieu, 1984, 1986), the availability of resources increases alternatives of action (dispositional flexibility) and preferences regarding the individual use expected. Resources comprise economic, cultural and social capital, which can be mutually transformed into one another (Bourdieu, 1986), as well as health capital, which is particularly important in old age (Grossman, 1972). All of these forms of capital are important for sports activities (Pampel et al., 2010; Studer et al., 2011; Eime et al., 2015; Hoebel et al., 2017; Jenkin et al., 2017). Regarding sports participation in older age, every resource has its individual limitations and is linked to specific incentive structures.

Economic capital (Bourdieu, 1986) is crucial when used for the provision of sport-promoting activities, such as sports equipment or fees for memberships (e.g. sports clubs, fitness studios). The economic capital that is used for other (leisure-time) activities and investment objects, such as travelling or financial support for other family members, can thus be deemed a decisive restriction. Furthermore, the availability of economic resources determines the place of residence of an individual, meaning that people with higher incomes enjoy greater opportunities to choose the place/region where they live. Especially in older age, when mobility can be limited, this factor is crucial for likelihood to participate in sports activities, as one may be forced to live in an area with infrastructural restrictions and a relatively low amount of opportunities to participate in sport and physical activities (Moschny et al., 2011).

Besides the influence of economic capital, Bourdieu (1986) discusses the importance of cultural capital, which can be converted into economic capital. Educational attainment $^{2}$ is positively related to embodied cultural capital, which includes knowledge about the individual health benefits of sports activities as well as a preference for sports participation as a useful vehicle for health promotion. Relatedly, with increasing knowledge about the positive effects of sports participation for health, the likelihood that individual resources will be directly invested in sports 
activities (health literacy) increases. It can be assumed that these resources determine the options available to participate in sport.

Furthermore, social capital is important when explaining sports participation. Social capital has direct and indirect effects on sports participation in general. On the one hand, with a larger number of social contacts, the alternatives available to act in the field of sports activities increase (Coleman, 1988). On the other hand, with an increase in 'specific' relations, access to information increases (Granovetter, 1973; Lin, 2001), which is strategically significant for sports activities, such as knowledge regarding current activity-related offerings for older people and access to professional actors of sports promotion in older age. Given that age is associated with a reduction in locally effective social relations (e.g. loss of social relations in the workplace, death of a spouse or friends), in the long term older adults are disadvantaged regarding access to sport and related opportunity structures and information. ${ }^{3}$ The extent to which this may be compensated by multiplex social relations is contingent on the value of sport within these social relations. Indeed, an individual's health behaviour may be influenced by the sports-related behaviour of his or her social relations (social contagion), as sports participation can increase social recognition by others. However, it can also work in the opposite direction where sport is perceived as inappropriate within the social group.

Regarding health/body capital, sports participation requires a certain level of physical constitution, but physical capacity decreases in older age (Heinemann, 1995; Robert Koch-Institut, 2015). According to the concept of health capital (Grossman, 1972), the initial individual stock of health capital decreases when getting old. Therefore, the age factor has an indicative function regarding individual health capital. The latter, therefore, represents a key restriction to the extent to which one can participate in sports. Inversely, individual health capital represents an important starting point for expected sports-related benefits. ${ }^{4}$ Through appropriate investments in sports activities, the reduction of individual health capital (decreasing physical ability) may be compensated or delayed.

According to the resource-based approach, differences (inequalities) regarding the level of sports participation seems plausible, but with limitations. Extending the simple resource-investment model, it is assumed that the (variable) use of available resources for sports activities is also significant. This implies that not only the availability of resources, but also their (variable) transformation in dependency to a particular health-related lifestyle may determine differences in older people's sports participation.

On the one hand, these inter-relationships may depend on the extent to which healthy ageing is prioritised. This means that attitudes regarding the importance of health and its proactive promotion determines the likelihood that economic capital will be invested to support measures such as healthy nutrition, preventive measures (e.g. health checks), and sports and other physical activities. On the other hand, evaluation of individual investments in sports activities might be relevant in terms of individual health promotion. Therefore, to what extent do older adults consider investments in sport or other kinds of physical activity an important strategy for their health and fitness? Nevertheless, it should be noted that older people are a heterogeneous population group and not all of them consider sport an effective medium for health and wellbeing, with some preferring relaxation (Dionigi and Gard, 2018). 
In addition to health-specific behaviour, age-specific attitudes should also be considered, as shown in specific age pictures. Age pictures can be understood as ageing-related opinions and beliefs that also suggest certain behavioural tendencies (Wurm et al., 2013). With the structural change of age (Robert-Koch Institut, 2009), altered norms and pictures of proactive ageing have been established. Therefore, older people interpret age as an independent phase of life, increasingly actively shaped by physical activities and sport (Thiel et al., 2008). Therefore, sports activities may be established in different ways in the everyday lives of older people, owing to variability in assessments and attitudes of health and age perception. It can consequently be assumed that the effects of economic, social and health resources on the sports participation of the older adults will decrease when healthspecific attitudes and age pictures are controlled.

In addition, it is essential to consider the fact that status affiliation is likely to have an impact on individual behaviour and attitudes. Physical capital or body experiences are determined by status-specific dispositions and habits as well as socio-cultural values (Bourdieu, 1978), and therefore, it can be assumed that health-related attitudes and activities of the older adults vary across different social status groups. Such specific differences in health-related attitudes and behaviour are empirically illustrated by the fact that people with lower socio-economic status tend to have more health-risky lifestyles (Gunasekara et al., 2011; Shaw et al., 2014). Therefore, the question arises of whether there exists status-related interrelationships of individual resources and health-promoting activities, such as sports activities in older age, an issue that has received little attention. Consequently, the extent to which such attitudes vary across different social status groups should be examined. If variations are small, it might be inferred that health-promoting activities are rather homogeneous (status crystallisation; see Lenski, 1966).

\section{Method}

\section{Data sample}

For our analysis, cross-sectional data from the fifth wave of the German Ageing Survey (released in 2014) were used. The German Ageing Survey is representative of the older German population and captures the life situation of people aged 4085. The questions asked cover (amongst other issues) health and health-related attitudes, behaviour, family and social networks, and economic situation.

For our analysis, a sub-sample was used containing only persons aged 65 years and over. Therefore, all persons included in the sample were no longer employed and could be assigned to old age (Martin and Kliegel, 2010; Destatis - Statistisches Bundesamt, 2017). Furthermore, to attain consistent results, only cases with information for each variable in the regression models were included $(\mathrm{N}=1,560)$.

\section{Measurement}

The dependent variable sports participation was captured by the question 'How often do you practise sport and exercise, for example hiking, playing football, gymnastics or swimming?' The possible response categories were $1=$ 'daily', 2 = 'several times per week', 3 ='once per week', $4=$ 'once to three times per month', 
$5=$ 'infrequently' and $6=$ 'never' (Deutsches Zentrum für Altersfragen, 2015). The categories were recoded $(1=$ 'never' to $6=$ 'daily').

Socio-demographic characteristics were represented by the variables gender $(0=$ 'male', $1=$ 'female'), age (in years) and marital status $(0=$ 'with partner', 1 = 'single', 2 = 'widowed').

Socio-economic resources were represented by the variables education ( $0=$ 'low', $1=$ 'middle', $2=$ 'high' $)^{5}$ and income poverty $(0=\text { 'no', } 1=\text { 'yes' })^{6}$ (economic and cultural capital). Social capital was represented by the frequency of meeting a solid group of people (meet solid group of people, 1 = 'daily to several times per week', 2 = 'several times per week to one to three times per month', 3 = 'infrequently/never') and the existence of persons who could be asked for advice ( persons for advice, $0=$ 'yes', $1=$ 'no'). Health capital was captured by self-rated health ( $1=$ 'very good', $2=$ 'good', $3=$ 'middle', 4 = 'bad', 5 = 'very bad') and chronic diseases ( $0=$ 'no', $1=$ 'yes, one', $2=$ 'yes, several').

The variable social status group categorised persons into three groups $(0=$ 'low social status', $1=$ 'middle social status', 2 ='upper social status') according to their actual or latest job position and the actual or latest job position of their spouse $^{7}$ (Mayer and Wagner, 1999; Kohli et al., 2000). Originally the variable consisted of five categories ( 1 ='underclass', 2 = 'lower-middle class', 3 = 'middlemiddle class', 4 = 'elevated-middle class', 5 = 'upper-middle class') (Deutsches Zentrum für Altersfragen, 2016a), but due to the small number of cases and potential problems regarding the analysis, the five categories were merged into the three aforementioned categories. Health-related behaviour was represented by health motivation ( $0=$ 'I do nothing', $5=$ 'I do a lot for it') and participating in health check-ups ( 0 = 'no', 1 = 'yes') (Deutsches Zentrum für Altersfragen, 2015).

Age perception was represented by three scales (sum scores). The first scale represented the individual's attitude regarding physical aspects in old age (age perception physical). For the development of the scale, three variables were used to query (among others) level of agreement with statements such as 'Age: not so strong anymore' and 'Age: less able to compensate for physical losses' (Deutsches Zentrum für Altersfragen, 2016b). ${ }^{8}$ A higher value here stated rejection of these statements. The scale was checked for reliability (Cronbach's $\alpha=0.74$ ). By capturing approval of four statements such as 'Ageing: continue to realise many ideas' and 'Ageing: expand my skills', the second scale represented the attitudes of the older adults regarding their individual skills (age perception skills) (Deutsches Zentrum für Altersfragen, 2016a). The variable was recoded, with a higher value stating agreement with these statements. The scale was checked for standardised polarity and reliability (Cronbach's $\alpha=0.71){ }^{9}$ The third scale represented a more future-related attitude regarding age by capturing approval of statements such as 'I look to the future with confidence' or 'For every problem I can find a solution' (future-related age perception) (Deutsches Zentrum für Altersfragen, 2016a). ${ }^{10} \mathrm{~A}$ higher value represented approval of the statements. The scale was checked for standardised polarity and reliability (Cronbach's $\alpha=0.76$ ).

\section{Analysis}

Due to scaling and absence of a normal distribution, a multiple Poisson regression was applied (Coxe et al., 2009; Kabacoff, 2015). Poisson regression is part of the 
family of generalised linear models, where the transformation of the predicted outcome is feasible, meaning that the predicted values of a dependent variable can be in a different metric than the observed values (Coxe et al., 2009).

In contrast to ordinary least squares (OLS) regression, Poisson regression uses the maximum likelihood estimator, akin to logistic regression. When $\ln (\hat{\mu})=b_{0}+$ $b_{1} X_{1}+b_{2} X_{2}+\ldots+b_{p} X_{p}$, our main model to explore the different effects of individual resources, health behaviour and age perception on sports participation in older age could be depicted as: $\ln ($ sports participation $)=b_{0}+b_{1}($ gender $)+b_{2}($ age $)+b_{3-}$ (marital status) $+b_{4}$ (education) $+b_{5}$ (income poverty $)+b_{6}$ (meet solid group of people $)+b_{7}$ (persons for advice $)+b_{8}$ (self-rated health $)+b_{9}$ (chronic diseases $)+b_{10-}$ (health motivation $)+b_{11}$ (health check-up) $+b_{12}$ (age perception physical $)+b_{13}$ (age perception skills $)+b_{14}$ (future-related age perception $)+\epsilon$.

First, sample characteristics differentiated according to sports participation are shown (Table 1). According to the scale level of the particular variables, frequencies (with percentage) and mean values (with standard deviation (SD)) are presented. Appropriate significance tests were conducted for all group-specific differences. According to the hypothesised varying effect structures regarding the different social status groups, sample characteristics are also stratified (Table 2). For the observation of the influences of predictive variables and their inter-relationships regarding sports participation in older age, the different variable groups are included stepwise in the analysis (Table 3). In Model 1 (socio-demographic characteristics), the effects of gender, age and marital status on sports participation are shown. Thereafter, socio-economic resources (education, income poverty), social capital (meet solid group of people, persons for advice) and health capital (self-rated health, chronic diseases), which are all deemed individual resources, are included. The variable groups health behaviour (health motivation, health check-up) and age perception (age perception physical, age perception skills, future-related age perception) were also included, focusing on behavioural and attitudinal aspects.

In order to explore differences regarding the inter-relationships between socioeconomic (individual) resources and age-related attitudes and behaviours on sports participation, three different regression models within the different social status groups were estimated (Table 4). Socio-economic resources were excluded here because assignment to a specific social status group was based on the latest job position, which correlates with the variables of education and income poverty, hence including these variables within the models may lead to multicollinearity and, therefore, biased results.

When using Poisson regression (and also logistic regression), the issue of overdispersion can occur, which means 'the observed variance of the response variable is larger than would be predicted by the Poisson regression' (Kabacoff, 2015: 315). If the ratio of the residual deviance to the residual degrees of freedom is much larger than 1, over-dispersion can exist (Kabacoff, 2015). All regression models were tested on over-dispersion and the ratio ranged from 0.79 to 1.14 , hence it can be assumed that over-dispersion was not a problem in our models. As indicators for the goodness of fit of the regression models, the pseudo- $R^{2}$ (Nagelkerke) - representing the 'proportional reduction in deviance due to the inclusion of the predictors' (Coxe et al., 2009: 126)-and the deviance goodness-of-fit test were applied. Furthermore, the regression models were tested 
Table 1. Sample characteristics stratified for sports participation

\begin{tabular}{|c|c|c|c|c|c|c|c|c|}
\hline & \multicolumn{7}{|c|}{ Sports participation } & \multirow[b]{2}{*}{$p^{1}$} \\
\hline & 1. Never & 2. Infrequent & $\begin{array}{l}\text { 3. Once to three times } \\
\text { per month }\end{array}$ & $\begin{array}{l}\text { 4. Once per } \\
\text { week }\end{array}$ & $\begin{array}{l}\text { 5. Several times } \\
\text { per week }\end{array}$ & 6. Daily & Total & \\
\hline $\mathrm{N}$ & 514 & 148 & 96 & 265 & 378 & 159 & & \\
\hline Gender (N, \%): & & & & & & & & $<0.01$ \\
\hline 0. Male & $287(0.33)$ & $104(0.12)$ & $62(0.07)$ & $112(0.13)$ & $224(0.26)$ & $82(0.09)$ & $871(0.56)$ & \\
\hline 1. Female & $227(0.33)$ & $44(0.06)$ & $34(0.05)$ & $153(0.23)$ & $154(0.23)$ & $77(0.11)$ & $689(0.44)$ & \\
\hline Age (M; SD) & $74.22(5.45)$ & $72.83(5.02)$ & $72.34(4.28)$ & $72.30(4.89)$ & $71.59(4.72)$ & $73.13(5.34)$ & $72.90(5.17)$ & $<0.01$ \\
\hline Marital status (N, \%): & & & & & & & & $<0.01$ \\
\hline 0. With partner & $331(0.30)$ & $115(0.10)$ & $75(0.07)$ & $182(0.17)$ & $293(0.27)$ & $107(0.10)$ & $1,103(0.71)$ & \\
\hline 1. Single & $63(0.33)$ & $15(0.08)$ & $8(0.04)$ & $35(0.19)$ & $42(0.22)$ & $26(0.14)$ & $189(0.12)$ & \\
\hline 2. Widowed & $120(0.45)$ & $18(0.07)$ & $13(0.05)$ & $48(0.18)$ & $43(0.16)$ & $26(0.10)$ & $268(0.17)$ & \\
\hline Education $(\mathrm{N}, \%)$ : & & & & & & & & $<0.01$ \\
\hline 0. Low & $79(0.56)$ & $12(0.09)$ & $4(0.03)$ & $19(0.14)$ & $16(0.11)$ & $10(0.07)$ & $140(0.09)$ & \\
\hline 1. Middle & $301(0.40)$ & $77(0.10)$ & $52(0.07)$ & $108(0.14)$ & $160(0.21)$ & $64(0.08)$ & $762(0.49)$ & \\
\hline 2. High & $134(0.20)$ & $59(0.09)$ & $40(0.06)$ & $138(0.21)$ & $202(0.31)$ & $85(0.13)$ & $658(0.42)$ & \\
\hline Income poverty (N, \%): & & & & & & & & $<0.01$ \\
\hline 0. No & $446(0.31)$ & $129(0.09)$ & $90(0.06)$ & $249(0.17)$ & $362(0.25)$ & $152(0.11)$ & $1,428(0.92)$ & \\
\hline 1. Yes & $68(0.52)$ & $19(0.14)$ & $6(0.05)$ & $16(0.12)$ & $16(0.12)$ & $7(0.05)$ & $132(0.09)$ & \\
\hline
\end{tabular}


Table 1. (Continued.)

\begin{tabular}{|c|c|c|c|c|c|c|c|c|}
\hline & \multicolumn{7}{|c|}{ Sports participation } & \multirow[b]{2}{*}{$p^{1}$} \\
\hline & 1. Never & 2. Infrequent & $\begin{array}{l}\text { 3. Once to three times } \\
\text { per month }\end{array}$ & $\begin{array}{l}\text { 4. Once per } \\
\text { week }\end{array}$ & $\begin{array}{l}\text { 5. Several times } \\
\text { per week }\end{array}$ & 6. Daily & Total & \\
\hline Social status group (N, \%): & & & & & & & & $<0.01$ \\
\hline 0. Low & $172(0.54)$ & $25(0.08)$ & $22(0.07)$ & $38(0.12)$ & $45(0.14)$ & $19(0.06)$ & $322(0.21)$ & \\
\hline 1. Middle & $283(0.31)$ & $99(0.11)$ & $55(0.06)$ & $159(0.17)$ & $229(0.25)$ & $93(0.10)$ & $918(0.59)$ & \\
\hline 2. Upper & $59(0.18)$ & $24(0.08)$ & $19(0.06)$ & $68(0.21)$ & $104(0.32)$ & $47(0.15)$ & $321(0.21)$ & \\
\hline $\begin{array}{l}\text { Meet solid group of people } \\
(\mathrm{N}, \%) \text { : }\end{array}$ & & & & & & & & $<0.01$ \\
\hline $\begin{array}{l}\text { 1. Daily to several times } \\
\text { per week }\end{array}$ & $29(0.21)$ & $9(0.07)$ & $4(0.03)$ & $26(0.19)$ & $55(0.40)$ & $14(0.10)$ & $137(0.09)$ & \\
\hline $\begin{array}{l}\text { 2. Once per week to } 1-3 \\
\text { times per month }\end{array}$ & $201(0.25)$ & $76(0.10)$ & $53(0.07)$ & $164(0.21)$ & $215(0.27)$ & $81(0.10)$ & $790(0.51)$ & \\
\hline 3. Infrequent/never & $284(0.45)$ & $63(0.10)$ & $39(0.06)$ & $75(0.12)$ & $108(0.17)$ & $64(0.10)$ & $633(0.41)$ & \\
\hline Persons for advice (N, \%): & & & & & & & & $<0.01$ \\
\hline 0. Yes & $440(0.31)$ & $136(0.10)$ & $88(0.06)$ & $247(0.17)$ & $363(0.26)$ & $148(0.10)$ & $1,422(0.91)$ & \\
\hline 1. No & $74(0.53)$ & $12(0.09)$ & $8(0.06)$ & $18(0.13)$ & $15(0.11)$ & $11(0.08)$ & $138(0.09)$ & \\
\hline Self-rated health $(\mathrm{N}, \%)$ : & & & & & & & & $<0.01$ \\
\hline 1. Very good & $14(0.14)$ & $2(0.02)$ & $4(0.04)$ & $15(0.16)$ & $46(0.48)$ & $16(0.17)$ & $97(0.06)$ & \\
\hline 2. Good & $172(0.25)$ & $60(0.09)$ & $59(0.09)$ & $130(0.19)$ & $187(0.27)$ & $77(0.11)$ & $685(0.44)$ & \\
\hline 3. Middle & $229(0.38)$ & $71(0.12)$ & $31(0.05)$ & $89(0.15)$ & $130(0.22)$ & $47(0.08)$ & $597(0.38)$ & \\
\hline 4. Bad & $82(0.51)$ & $15(0.09)$ & $2(0.01)$ & $31(0.19)$ & $15(0.09)$ & $16(0.10)$ & $161(0.10)$ & \\
\hline 5. Very bad & $17(0.85)$ & $0(0.00)$ & $0(0.00)$ & $0(0.00)$ & $0(0.00)$ & $3(0.15)$ & $20(0.01)$ & \\
\hline
\end{tabular}


Chronic diseases (N, \%):

\begin{tabular}{|c|c|c|c|c|c|c|c|c|}
\hline 0. No & $155(0.27)$ & $57(0.10)$ & $41(0.07)$ & $97(0.17)$ & $170(0.29)$ & $58(0.10)$ & $578(0.37)$ & \\
\hline 1. Yes, one & $201(0.34)$ & $56(0.10)$ & $39(0.07)$ & $98(0.17)$ & $134(0.23)$ & $56(0.10)$ & $584(0.37)$ & \\
\hline 2. Yes, several & $158(0.40)$ & $35(0.09)$ & $16(0.04)$ & $70(0.18)$ & $74(0.19)$ & $45(0.11)$ & $398(0.26)$ & \\
\hline Health motivation (M; SD) & $3.96(1.21)$ & $4.01(1.10)$ & $4.10(0.93)$ & $4.03(1.03)$ & $4.29(0.87)$ & $4.43(0.85)$ & $4.12(1.05)$ & $<0.01$ \\
\hline Health check-up (N, \%): & & & & & & & & $<0.01$ \\
\hline 0. No & $203(0.41)$ & $61(0.12)$ & $25(0.05)$ & $81(0.16)$ & $86(0.17)$ & $45(0.09)$ & $501(0.32)$ & \\
\hline 1. Yes & $311(0.29)$ & $87(0.08)$ & $71(0.07)$ & $184(0.17)$ & $292(0.28)$ & $114(0.11)$ & $1,059(0.68)$ & \\
\hline $\begin{array}{l}\text { Age perception physical } \\
\text { (M; SD) }\end{array}$ & $2.02(0.55)$ & $2.16(0.52)$ & $2.16(0.49)$ & $2.21(0.57)$ & $2.34(0.55)$ & $2.25(0.60)$ & $2.17(0.41)$ & $<0.01$ \\
\hline Age perception skills (M; SD) & $2.64(0.62)$ & $2.89(0.55)$ & $2.95(0.51)$ & $2.84(0.56)$ & $3.01(0.51)$ & $2.95(0.52)$ & $2.84(0.44)$ & $<0.01$ \\
\hline $\begin{array}{l}\text { Future-related age } \\
\text { perception (M; SD) }\end{array}$ & $3.02(0.48)$ & $3.10(0.47)$ & $3.18(0.35)$ & $3.04(0.45)$ & $3.17(0.38)$ & $3.14(0.41)$ & $3.09(0.44)$ & $<0.01$ \\
\hline
\end{tabular}

Notes: $\mathrm{N}=1,560 . \mathrm{M}=$ mean. $\mathrm{SD}$ : standard deviation. $1 . \chi^{2}$-test/Kruskal-Wallis test.

Source: German Ageing Survey, 2014. 


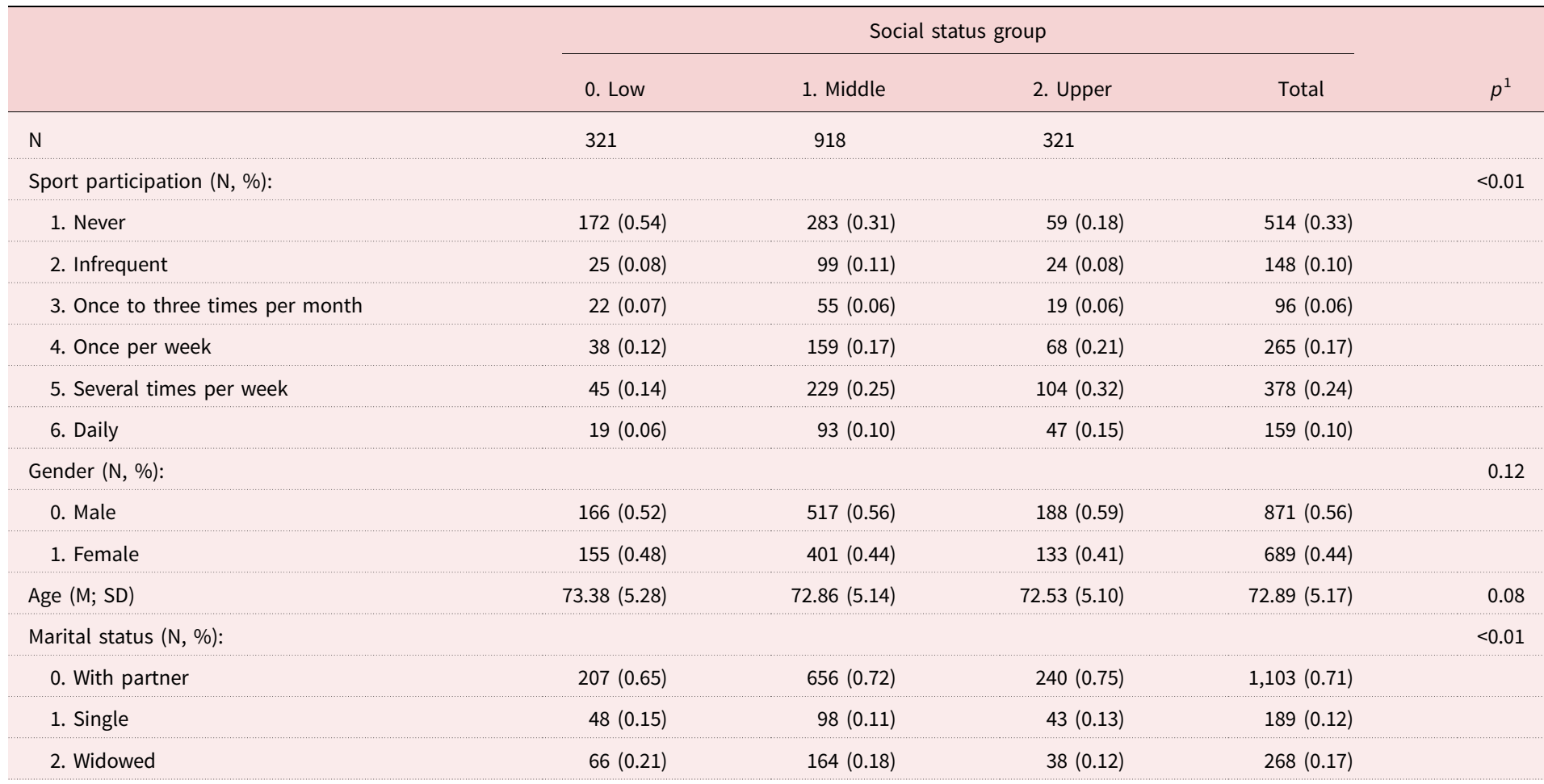




\begin{tabular}{|c|c|c|c|c|c|}
\hline 0. Low & $64(0.20)$ & $67(0.07)$ & $9(0.03)$ & $140(0.09)$ & \\
\hline 1. Middle & $214(0.67)$ & $461(0.50)$ & $87(0.27)$ & $762(0.49)$ & \\
\hline 2. High & $43(0.13)$ & $390(0.43)$ & $225(0.70)$ & $658(0.42)$ & \\
\hline Income poverty (N, \%): & & & & & $<0.01$ \\
\hline 0. No & $264(0.82)$ & $856(0.93)$ & $308(0.96)$ & $1,428(0.92)$ & \\
\hline 1. Yes & $57(0.18)$ & $62(0.07)$ & $13(0.04)$ & $132(0.09)$ & \\
\hline Meet solid group of people (N, \%): & & & & & $<0.01$ \\
\hline 1. Daily to several times per week & $28(0.09)$ & $82(0.09)$ & $27(0.08)$ & $137(0.09)$ & \\
\hline 2. Once per week to $1-3$ times per month & $126(0.39)$ & $487(0.53)$ & $177(0.55)$ & $790(0.51)$ & \\
\hline 3. Infrequent/never & $167(0.52)$ & $349(0.38)$ & $117(0.36)$ & $633(0.41)$ & \\
\hline Persons for advice (N, \%): & & & & & $<0.05$ \\
\hline 0. Yes & $280(0.87)$ & $841(0.92)$ & $301(0.94)$ & $1,422(0.91)$ & \\
\hline 1. No & $41(0.13)$ & $77(0.08)$ & $20(0.06)$ & $138(0.09)$ & \\
\hline Self-rated health $(\mathrm{N}, \%)$ : & & & & & $<0.01$ \\
\hline 1. Very good & $8(0.03)$ & $50(0.05)$ & $39(0.12)$ & $97(0.06)$ & \\
\hline 2. Good & $115(0.36)$ & $421(0.46)$ & $149(0.46)$ & $685(0.44)$ & \\
\hline 3. Middle & $150(0.47)$ & $343(0.37)$ & $104(0.32)$ & $597(0.38)$ & \\
\hline 4. Bad & $43(0.13)$ & $92(0.10)$ & $26(0.08)$ & $161(0.10)$ & \\
\hline 5. Very bad & $5(0.02)$ & $12(0.01)$ & $3(0.01)$ & $20(0.01)$ & \\
\hline Chronic diseases $(\mathrm{N}, \%)$ : & & & & & 0.12 \\
\hline 0. No & $114(0.36)$ & $333(0.36)$ & $131(0.41)$ & $578(0.37)$ & \\
\hline 1. Yes, one & $111(0.35)$ & $363(0.40)$ & $110(0.34)$ & $584(0.37)$ & \\
\hline 2. Yes, several & $96(0.30)$ & $222(0.24)$ & $80(0.25)$ & $498(0.26)$ & \\
\hline
\end{tabular}


Table 3. Poisson regression: determinants of sports participation in older age (between-model (stepwise))

\begin{tabular}{|c|c|c|c|c|c|c|}
\hline & $\begin{array}{l}\text { Model 1: } \\
\text { Socio-demographic } \\
\text { characteristics }\end{array}$ & $\begin{array}{l}\text { Model 2: } \\
\text { Socio-economic } \\
\text { resources }\end{array}$ & $\begin{array}{c}\text { Model 3: } \\
\text { Social capital }\end{array}$ & $\begin{array}{c}\text { Model 4: } \\
\text { Health capital }\end{array}$ & $\begin{array}{l}\text { Model 5: } \\
\text { Health } \\
\text { behaviour }\end{array}$ & $\begin{array}{c}\text { Model 6: } \\
\text { Age perception }\end{array}$ \\
\hline Intercept & $2.35^{\star \star \star}(0.21)$ & $1.87^{\star \star \star}(0.23)$ & $1.86^{\star \star \star}(0.23)$ & $1.96^{\star \star \star}(0.23)$ & $1.54^{\star \star \star}(0.24)$ & $0.86^{\star \star}(0.30)$ \\
\hline \multicolumn{7}{|l|}{ Gender } \\
\hline 0. Male & Ref. & Ref. & Ref. & Ref. & Ref. & Ref. \\
\hline 1. Female & $0.06^{\star}(0.03)$ & $0.13^{\star \star \star}(0.03)$ & $0.11^{\star \star \star}(0.03)$ & $0.11^{\star \star \star}(0.03)$ & $0.09^{\star \star}(0.03)$ & $0.09^{\star \star}(0.03)$ \\
\hline Age & $-0.02^{\star \star \star}(0.00)$ & $-0.01^{\star \star \star}(0.00)$ & $-0.01^{\star \star \star}(0.00)$ & $-0.01^{\star \star}(0.00)$ & $-0.01^{\star \star \star}(0.00)$ & $-0.01^{\star \star}(0.00)$ \\
\hline \multicolumn{7}{|l|}{ Marital status: } \\
\hline 0. With partner & Ref. & Ref. & Ref. & Ref. & Ref. & Ref. \\
\hline 1. Single & $-0.01(0.04)$ & $0.02(0.04)$ & $0.03(0.04)$ & $0.03(0.04)$ & $0.05(0.04)$ & $0.05(0.04)$ \\
\hline 2. Widowed & $-0.12^{\star}(0.04)$ & $-0.07(0.04)$ & $-0.07(0.04)$ & $-0.06(0.04)$ & $-0.04(0.04)$ & $-0.04(0.04)$ \\
\hline \multicolumn{7}{|l|}{ Education: } \\
\hline 0. Low & & Ref. & Ref. & Ref. & Ref. & Ref. \\
\hline 1. Middle & & $0.17^{\star \star}(0.06)$ & $0.18^{\star \star}(0.06)$ & $0.16^{\star \star}(0.06)$ & $0.15^{\star}(0.06)$ & $0.14^{\star}(0.06)$ \\
\hline 2. High & & $0.41^{\star \star \star}(0.06)$ & $0.40^{\star \star \star}(0.06)$ & $0.37^{\star \star \star}(0.06)$ & $0.38^{\star \star \star}(0.06)$ & $0.36^{\star \star \star}(0.06)$ \\
\hline \multicolumn{7}{|l|}{ Income poverty: } \\
\hline 0. No & & Ref. & Ref. & Ref. & Ref. & Ref. \\
\hline 1. Yes & & $-0.26^{\star \star \star}(0.06)$ & $-0.24^{\star \star \star}(0.06)$ & $-0.20^{\star \star}(0.06)$ & $-0.19^{\star \star}(0.06)$ & $-0.18^{\star *}(0.06)$ \\
\hline
\end{tabular}


Table 3. (Continued.)

\begin{tabular}{|c|c|c|c|c|c|c|}
\hline & $\begin{array}{l}\text { Model 1: } \\
\text { Socio-demographic } \\
\text { characteristics }\end{array}$ & $\begin{array}{l}\text { Model 2: } \\
\text { Socio-economic } \\
\text { resources }\end{array}$ & $\begin{array}{c}\text { Model 3: } \\
\text { Social capital }\end{array}$ & $\begin{array}{c}\text { Model 4: } \\
\text { Health capital }\end{array}$ & $\begin{array}{l}\text { Model } 5 \text { : } \\
\text { Health } \\
\text { behaviour }\end{array}$ & $\begin{array}{c}\text { Model 6: } \\
\text { Age perception }\end{array}$ \\
\hline \multicolumn{7}{|l|}{ Meet solid group of people: } \\
\hline 1. Daily to several times per week & & & Ref. & Ref. & Ref. & Ref. \\
\hline 2. Once per week to $1-3$ times per month & & & $-0.09^{\star}(0.05)$ & $-0.09(0.05)$ & $-0.09(0.05)$ & $-0.08(0.05)$ \\
\hline 3. Infrequent/never & & & $-0.26^{\star \star \star}(0.05)$ & $-0.24^{\star \star \star}(0.05)$ & $-0.22^{\star \star \star}(0.05)$ & $-0.21^{\star \star \star}(0.05)$ \\
\hline 0 . Yes & & & Ref. & Ref. & Ref. & Ref. \\
\hline 1. No & & & $-0.21^{\star \star \star}(0.06)$ & $-0.19^{\star \star \star}(0.06)$ & $-0.19^{\star \star}(0.06)$ & $-0.17^{\star \star}(0.06)$ \\
\hline \multicolumn{7}{|l|}{ Self-rated health: } \\
\hline 1. Very good & & & & Ref. & Ref. & Ref. \\
\hline \multicolumn{7}{|l|}{ Chronic diseases: } \\
\hline 0. No & & & & Ref. & Ref. & Ref. \\
\hline 1. Yes, one & & & & $-0.01(0.03)$ & $-0.03(0.03)$ & $-0.02(0.03)$ \\
\hline 2. Yes, several & & & & $0.01(0.04)$ & $0.00(0.04)$ & $0.01(0.04)$ \\
\hline Health motivation & & & & & $0.10^{\star \star \star}(0.01)$ & $0.10^{\star \star \star}(0.01)$ \\
\hline
\end{tabular}




\section{Health check-up:}

\begin{tabular}{|c|c|c|c|c|c|c|}
\hline 0. No & & & & & Ref. & Ref. \\
\hline 1. Yes & & & & & $0.12^{\star \star \star}(0.03)$ & $0.12^{\star}(0.03)$ \\
\hline Age perception physical & & & & & & $0.08^{\star \star}(0.03)$ \\
\hline Age perception skills & & & & & & $0.10^{\star \star}(0.03)$ \\
\hline Future-related age perception & & & & & & $-0.13^{\star \star}(0.04)$ \\
\hline N & 1,560 & 1,560 & 1,560 & 1,560 & 1,560 & 1,560 \\
\hline Nagelkerke- $R^{2}$ & 0.049 & 0.153 & 0.203 & 0.240 & 0.287 & 0.304 \\
\hline $\begin{array}{l}\text { Deviance goodness } \\
\text { of fit }(p)\end{array}$ & 0.00 & 0.04 & 0.21 & 0.48 & 0.85 & 0.92 \\
\hline
\end{tabular}

Notes: $B$-coefficients (standard errors in parentheses). Ref.: reference category.

Source: German Ageing Survey, 2014.

Significance levels: ${ }^{\star} p<0.05,{ }^{\star \star} p<0.01,{ }^{\star \star *} p<0.001$. 
Table 4. Multiple Poisson regression: determinants of sports participation in older age (within-models)

\begin{tabular}{|c|c|c|c|}
\hline & $\begin{array}{l}\text { Model } 1 \text { : } \\
0 . \text { Low social } \\
\text { status }\end{array}$ & $\begin{array}{l}\text { Model 2: } \\
\text { 1. Middle social } \\
\text { status }\end{array}$ & $\begin{array}{l}\text { Model 3: } \\
\text { 2. Upper social } \\
\text { status }\end{array}$ \\
\hline Intercept & $1.50^{\star}(0.70)$ & $1.37^{\star \star \star}(0.36)$ & $2.21^{\star \star \star}(0.55)$ \\
\hline \multicolumn{4}{|l|}{ Gender: } \\
\hline 0. Male & Ref. & Ref. & Ref. \\
\hline 1. Female & $0.04(0.08)$ & $0.03(0.04)$ & $0.00(0.06)$ \\
\hline Age & $-0.01(0.01)$ & $-0.01(0.00)$ & $-0.02^{\star \star}(0.01)$ \\
\hline \multicolumn{4}{|l|}{ Marital status: } \\
\hline 0. With partner & Ref. & Ref. & Ref. \\
\hline 1. Single & $-0.04(0.11)$ & $0.05(0.06)$ & $0.05(0.08)$ \\
\hline 2. Widowed & $-0.06(0.10)$ & $-0.08(0.05)$ & $-0.01(0.10)$ \\
\hline \multicolumn{4}{|l|}{ Meet solid group of people: } \\
\hline $\begin{array}{l}\text { 1. Daily to several times } \\
\text { per week }\end{array}$ & Ref. & Ref. & Ref. \\
\hline $\begin{array}{l}\text { 2. Once per week to } 1-3 \\
\text { times per month }\end{array}$ & $-0.07(0.12)$ & $-0.10(0.06)$ & $-0.08(0.10)$ \\
\hline 3. Infrequent/never & $-0.26^{\star}(0.13)$ & $-0.23^{\star \star \star}(0.07)$ & $-0.16(0.11)$ \\
\hline \multicolumn{4}{|l|}{ Persons for advice: } \\
\hline 0. Yes & Ref. & Ref. & Ref. \\
\hline 1. No & $-0.11(0.13)$ & $-0.15^{\star}(0.08)$ & $-0.17(0.13)$ \\
\hline \multicolumn{4}{|l|}{ Self-rated health: } \\
\hline 1. Very good & Ref. & Ref. & Ref. \\
\hline 2. Good & $0.02(0.21)$ & $-0.08(0.08)$ & $-0.19 *(0.09)$ \\
\hline 3. Middle & $-0.10(0.22)$ & $-0.21^{\star}(0.08)$ & $-0.18(0.10)$ \\
\hline 4. Bad & $-0.22(0.25)$ & $-0.23^{\star}(0.10)$ & $-0.36^{\star}(0.16)$ \\
\hline 5. Very bad & $-0.86(0.52)$ & $-0.36(0.21)$ & $-1.45^{*}(0.60)$ \\
\hline \multicolumn{4}{|l|}{ Chronic diseases: } \\
\hline 0. No & Ref. & Ref. & Ref. \\
\hline 1. Yes, one & $-0.11(0.09)$ & $-0.03(0.04)$ & $0.05(0.07)$ \\
\hline 2. Yes, several & $-0.02(0.10)$ & $0.01(0.05)$ & $0.06(0.09)$ \\
\hline Health motivation & $0.16^{\star \star \star}(0.04)$ & $0.07^{\star \star \star}(0.02)$ & $0.10^{\star \star \star}(0.03)$ \\
\hline \multicolumn{4}{|l|}{ Health check-up: } \\
\hline 0. No & Ref. & Ref. & Ref. \\
\hline 1. Yes & $0.19 *(0.08)$ & $0.15^{\star \star \star}(0.04)$ & $0.07(0.06)$ \\
\hline Age perception physical & $0.08(0.17)$ & $0.08^{\star}(0.04)$ & $0.02(0.06)$ \\
\hline Age perception skills & $0.22^{\star \star}(0.09)$ & $0.07(0.04)$ & $0.09(0.07)$ \\
\hline
\end{tabular}


Table 4. (Continued.)

\begin{tabular}{lccc}
\hline & $\begin{array}{c}\text { Model 1: } \\
\text { 0. Low social } \\
\text { status }\end{array}$ & $\begin{array}{c}\text { Model 2: } \\
\text { 1. Middle social } \\
\text { status }\end{array}$ & $\begin{array}{c}\text { Model 3: } \\
\text { 2. Upper social } \\
\text { status }\end{array}$ \\
\hline Future-related age perception & $-0.38^{\star \star \star}(0.09)$ & $-0.07(0.05)$ & $-0.06(0.09)$ \\
$\mathrm{N}$ & 321 & 918 & 321 \\
\hline Nagelkerke- $R^{2}$ & 0.349 & 0.197 & 0.263 \\
\hline $\begin{array}{l}\text { Deviance goodness of fit } \\
(p \text { value })\end{array}$ & 0.57 & 0.35 & 1.00 \\
\hline
\end{tabular}

Notes: $B$-coefficients (standard errors in parentheses). Ref.: reference category.

Source: German Ageing Survey, 2014.

Significance levels: ${ }^{\star} p<0.05,{ }^{\star *} p<0.01,{ }^{\star \star *} p<0.001$.

for multicollinearity (variance inflation factor). For analyses, Microsoft R Open 3.5.0 (RStudio 1.1.463) with the packages 'foreign', 'texreg', 'Hmisc', 'car', 'haven', 'gmodels', 'sjstats', 'plm' and 'qcc' was used. ${ }^{11}$

\section{Results}

\section{Descriptive findings}

In Table 1, the sample characteristics stratified for sports participation are presented. Sixty-five per cent of the sample are male, with a mean age of 73 $(\mathrm{SD}=5.17)$; moreover, 71 per cent of the sample are married and living in a partnership. Ninety-one per cent of the sample claim to have a middle or high level of education and only 9 per cent are affected by income poverty. The largest part of the sample (59\%) can be assigned to the middle social status. Fifty-one per cent meet a solid group of people between once per week and three times per month, and 91 per cent claim to have a person for advice. Most of the participating persons declare good health (44\%) and 26 per cent claim to have several chronic diseases. Health motivation is relatively high $($ mean $=4.12, \mathrm{SD}=1.05)$ and 68 per cent participate in health check-ups. Age perception physical has a mean of 2.17 $(\mathrm{SD}=0.41)$, age perception skills $2.84(\mathrm{SD}=0.44)$ and future-related age perception $3.09(\mathrm{SD}=0.44)$. In the male and female groups, 33 per cent do not participate in sport, overwhelmingly the oldest people in the sample (mean $=74.22, \mathrm{SD}=5.45$ ). Furthermore, within the widowed group most people claim not to participate in sports (45\%). The highest proportion of non-participation in sports can be found in the low-education group (56\%), and this also applies to those in income poverty (52\%) and of low social status (54\%). Among the group of people who claim to meet a solid group infrequently or never (45\%), as well as those with no person for advice (53\%), most never participate in sports. The same applies to the group of people with bad (51\%) or very bad (85\%) self-rated health and people who declare themselves as having several chronic diseases (40\%). People with the lowest mean health motivation (mean $=3.96, \mathrm{SD}=1.21$ ) do not participate in sports; the same applies to the group of people who claim not to participate in health check-ups (41\%). Finally, the group of people who do not participate 
in sports also display the lowest mean values in age perception physical (mean = $2.02, \mathrm{SD}=0.55)$, age perception skills $($ mean $=2.64, \mathrm{SD}=0.62)$ and future-related age perception $($ mean $=3.02, \mathrm{SD}=0.48)$.

In Table 2, differences within the different social status groups are shown. In comparison to the other status groups, the proportion of people who never participate in sports is highest in the lowest social status group (54\%). The proportion of widowed people is also higher in the lowest status group. Furthermore, differences regarding social capital can be identified. In the lowest social status group, the percentage of people who meet a solid group of people only infrequently or never is highest (52\%), and this is also true of the percentage of people with no person for advice (13\%). When comparing health motivation across the social status groups, a higher value can be found for the lowest status group (mean $=4.20$, $\mathrm{SD}=1.10$ ) and the lowest value is in the highest status group (mean $=4.02$, $\mathrm{SD}=1.04)$. The lowest values of age perception physical (mean $=2.09, \mathrm{SD}=0.58)$, age perception skills (mean $=2.68, \mathrm{SD}=0.62$ ) and future-related age perception (mean $=3.02, \mathrm{SD}=0.48)$ can be found in the lowest social status group.

\section{Multiple analysis findings}

In Table 3, the results of the multiple Poisson regression are presented, applied in order to predict sports participation in older age. In Model 1 a positive effect of being female $(b=0.06, p<0.05)$ and a negative effect of age $(b=-0.02, p<0.05)$ on sports participation is evident. Being widowed is also negatively associated with sports participation $(b=-0.12, p<0.05)$. When including the socio-economic variables education and income poverty in Model 2, a positive effect of education (category high: $b=0.41, p<0.05$ ) and a negative effect of income poverty $(b=-0.26, p<0.05)$ can be seen. After including socio-economic resources, the effects of gender and age remain significant, but the effect of marital status loses its significance. In Model 3 (social capital), meeting a solid group of people only infrequently or never $(b=-0.26, p<0.05)$ and not having a person for advice $(b=-0.21, p<0.05)$ are negatively associated with sports participation. Therefore, a positive effect of social relations on sports participation can be confirmed. A slight decrease in the effect of the socio-demographic and economic variables is found (partial mediation). In Model 4, an effect of self-rated health is found. Inferior self-rated health is negatively associated with sports participation (very bad: $b=-0.63, p<0.05$ ), but no effect of chronic diseases can be identified. Furthermore, a slight decrease in the effects of the previously included variables is found. Positive health motivation $(b=0.10, p<0.05)$ and participating in regular health check-ups $(b=0.12, p<0.05)$ are positively associated with sports participation. Despite the effect of education, further slight decreases in the effects of the other variables can be noted. Age perception physical $(b=0.08, p<0.05)$ and age perception skills $(b=0.10, p<0.05)$ are positively associated with sports participation. However, future-related age perception $(b=-0.13, p<0.05)$ is negatively associated. It can be stated that although the inclusion of variables representing health behaviour and age perception result in minor decreases in the effects of the socioeconomic resources, these effects are still significant (partial mediation). It becomes clear that both socio-economic variables and the subjective measurements of health 
behaviour and age-specific attitudes have strong and statistically significant effects. There are only slight changes in the effects of the socio-economic variables compared to the previous models after including the subjective measurements. Thus, it becomes apparent that individual resources and attitude-related variables do not share covariance and each affect sports participation separately. Furthermore, the block-wise inclusion of variables also clarifies the increase in the model's fit. Having included all variable groups, 30.4 per cent of the variance in sports participation can be explained by the applied model.

To clarify the extent to which the effects of the applied model explaining sports participation in older age vary within the three social status groups, separate models were estimated. The central result of these models is that the significant predictors do not point in different directions; however, the influencing power of individual resources and attitude-related variables differ within the status group-specific models.

First, no effect of gender is found, but age is negatively associated with sports participation in Model 3 (upper social status). In none of the three different models is any effect of marital status identified. No effect of social capital on sports participation can be found in the model of the upper social status. In Model 1 (low social status), no effect of self-rated health is found. The variable chronic diseases does not affect sports participation in any of the three models. Health behaviour affects sports participation all three models, except for the variable health check-up in Model 3. Only for the category 'middle social status' (Model 2) is an effect of the variable age perception physical found. Age perception skills and future-related age perception are only significant determinants of sports participation in the low social status group (Model 1).

Finally, differences regarding the model's fit can be stated. Due to the higher pseudo- $R^{2}$ in Model $1(0.349)$, it can be assumed that the applied model fits and describes sports participation in older age more effectively for people belonging to the lowest of the three social status groups, as greater variance in the variable sports participation can be explained. It can be concluded that for the lowest social status group (and contrary to the other social status groups) health behaviour and age perception, in particular, are more significant in influencing sports participation in older age. Due to the higher standard errors in Model 1, it can be assumed that the lowest social status group is characterised by a higher variance. Thus, the differences suggest that older people with lower social status exhibit greater diversity in terms of their health behaviour and age perception, which accompanies increased effectiveness in sports participation. On the other hand, among older people with higher social status, health-related behaviour and age perception are at a superior level and more similar. Therefore, the differences regarding the explanation of sports participation are less relevant.

\section{Discussion}

This paper has sought to explain sports participation in older age, while integrating both traditional factors of social inequality (individual resources) and more subjective factors such as health behaviour and age-specific perceptions, in order to develop a broader picture of the inter-relationships and potential mediations 
between the variables. Research with a similar approach within this context remains rather scarce, but a deeper understanding of the inter-relationships between these variables as well as potential differences in effect structures regarding the different social status groups is required in order to develop more effective policies to promote sports participation in ageing societies.

Merely using individual resources or other vertical and horizontal factors of social inequalities is insufficient to explain sports participation in older age, which can be traced back to, among others, the process of individualisation and corresponding developments (Beck, 1994). The aim of our study was to analyse the potential inter-relationships between individual resources and the age-specific behaviours and attitudes that affect sports participation in older age. Given that sociological research on social inequalities has also confirmed the effect of affiliation to a specific social status group on behaviour and attitudes (social class mentality) (Lenski, 1966; Bourdieu, 1984), differences in the effect structures within different social status groups were analysed.

In line with previous research, our findings highlight a positive relationship between individual resources and sports participation, which means that people with (for instance) greater educational attainment or a superior stock of health capital are more likely to participate in sports (social gradient). Regarding health capital, a positive effect of self-rated health (but not objective health) can be seen. This may be explained by findings from other studies that show, on the one hand, a growing discrepancy between subjective and objective health in older age, but on the other hand, the fact that in old age, self-rated health may prove more important in predicting mortality and wellbeing (Idler and Benyamini, 1997; Szybalska et al., 2018). The positive effect of health behaviour on sports participation can be traced back to the specific motivations of the older adults to participate in sports and physical activities. Given that the health-promoting effect of sport represents a key motivation for sports participation in older age (Lehnert et al., 2012), it is likely that people who already have a healthy lifestyle (including regular health checkups) participate more regularly in sport because they are more aware of its health benefits. Regarding the positive effect of age perception (physical and skills) on sports participation, it is likely that the optimistic older adults seek to maintain their health and their mobility, and are aware of the importance of sport and physical activities (or active ageing in general) for the maintenance of mobility, autonomy and wellbeing. The negative relationship between future-related age perception and sports participation can be traced back to the fact that especially those people who currently have a negative attitude towards age and their future want to alter their present situation (or health and health behaviour) by increasing their participation in sports activities (as a kind of compensation strategy), in order to ensure a healthier future for themselves. Given that there is only a slight mediation effect in individual resources (having included variables depicting health- and age-specific behaviour and attitudes) and the different variable groups remain significant, only slight inter-relationships can be confirmed. Therefore, it can be concluded that individual resources, health behaviour and age-specific attitudes are independent determinants of sports participation in older age. This is of considerable relevance for the development of future prevention programmes to promote sports activities in older age, because it is not only necessary to improve socio-economic 
conditions (e.g. income, investment in education, and so forth) and improve opportunity structures (e.g. more offers and opportunities to participate in sport, infrastructural conditions) in order to enable older people to participate in sport (Del Castillo et al., 2010). Indeed, behavioural or attitudinal changes are also important and should be addressed by health-promoting policies in a more specific manner. Preventive actions could be developed, assisting in providing information about the importance of sports activities for health, so that older people become more aware of the potential health risks of inactivity. Such increased awareness can affect intentions to amend health-related behaviours ${ }^{12}$ (Arnautovska et al., 2018). Furthermore, efforts should be made to create (socio-cultural) general conditions that enable older people to change their inactive behaviour and/or maintain their sports activities. Given that people are influenced by behaviour in their social surroundings (social contagion), age-specific meetings could be organised where both inactive as well as active older people can share their experiences regarding the relative benefits of health-promoting behaviour over other age-specific daily activities. A further important aspect regarding the development of preventive programmes, even though it was not part of our analysis, is older adults' (individual) experiences of participating in current or past sports programmes (Griffin, 2017); meaning that, how a person has perceived participation in sports programmes, e.g. regarding the structure/organisation and accessibility, as well as social aspects of those sports courses, is crucial (Phoenix and Bell, 2019). A further important point that could help to increase participation rates in older age is to focus on possible joy and pleasure from these programmes, rather than sports or physical activity as a medium for longevity and healthy ageing (Phoenix and Orr, 2014). Here it should also be noted that lived experiences as well as individual body experiences can also be shaped by status-specific dispositions and socio-cultural values (Bourdieu, 1978; Tulle and Dorrer, 2012).

Furthermore, discussion is necessary regarding the extent to which health- and age-specific attitudes are decoupled from status-specific influences. According to this study's findings, there is no clear response in one direction or the other. Health- and age-specific attitudes are significant for sports participation in the lower social status group. Additionally, more variability within this status group can be assumed. On the other hand, less variability is found in the other two social status groups, implying greater homogeneity in terms of health- and age-specific attitudes within these status groups. This suggests that health- and age-specific attitudes continue to indicate status-specific differences. Bourdieu's (1978) work on physical capital supports this argumentation since he pointed out that, aside from individual decisions, physical capital or body experiences are also shaped by status-specific dispositions and habits, as well as socio-cultural values, which could explain the influence of behavioural variables in our models. Therefore, status-specific and socio-cultural characteristics should be considered when designing sports programmes for older adults, especially regarding physicality and movement.

Given that age perception skills and future-related age perception are only significant determinants of sports participation in the lowest social status group, health policies focusing on age-specific attitudes in order to change health-specific behaviours such as sports activities should especially address older people belonging to the lowest social status group. This leads to the assumption that in the lowest social 
status group, age-specific attitudes in particular ought to be addressed by health policies focusing on the promotion of sports participation in older age. Physical capital (specific body-related and socio-cultural valued attributes, e.g. not looking too old, being in good shape, etc.) is more likely to be represented in the upper social status group and has a distinguishing character (Bourdieu, 1978). This could help to explain why age perception is significant in the lowest social status group, since physical capital could be used to overcome one's own social background (maybe in older age, there is a greater need for distinction in the lowest social status group); or conversely reflected, since the motivation of participating in sports differs regarding different social status groups (over the lifecourse) (e.g. 'body for others' and more health-oriented in upper social status group; Bourdieu, 1978: 834), one may argue that the older adults in upper social status groups do not need to distinguish themselves anymore and specific age perceptions are not crucial for participating in sports for this group. Furthermore, the individual experiences of one's own body as well as specific perceptions of ageing may vary regarding the different social status groups and individual dispositions (Bourdieu, 1978; Tulle and Dorrer, 2012). Since age perception is least positive in the lower social status group (see Table 2), it can be assumed that the association of ageing with a decline of physical abilities is more represented in the lowest social status group (that means positive body experiences could be less represented) which may explain the significant effect of age perception in the lowest social status group. However, it should also be considered that there are people in the lowest social status group who already possess these attitudes. Therefore, healthpromoting actions and programmes should be designed more differentially in order to prove more attractive and effective as well as to increase compliance within the target group.

In comparison to other studies, our simultaneous consideration of the two perspectives - individual resources and age- and health-related attitudes - in the model of sports participation of older people helps improve the explained variance of the estimated regression models. Nevertheless, it is also important to indicate some limitations when interpreting the findings. (a) Sports participation is based on the self-assessment of the older adults and should therefore be interpreted with caution. Furthermore, it is not possible to distinguish the kind of sport in which the older people surveyed participate, yet such information is important in identifying motivation since choosing a specific kind of sport is also determined by the affiliation to a specific social status group (Bourdieu, 1978). (b) According to an inter-temporal perspective, health- and age-specific attitudes (continuation, maintaining or resuming certain activities or investments of available resources) simultaneously represent reactions to previous experiences and adjustments to potential (failed) developments. Thus, further explanatory potential can be seen in the fact that corresponding adaptation and compensation strategies are taken in the sense of inter-temporal allocation decisions. However, analysis of such dynamic cause-effect relationships across the lifecycle requires longitudinal data. (c) Furthermore, consideration of both the individual aspects of older people as well as their corresponding contextual conditions (infrastructural restrictions, access to public transport, housing conditions, and so on) should help explain older people's sports participation, necessitating multi-level analysis. (d) Finally, 
limitations exist when distinguishing by social status group. Assignment here was based on the actual or latest job position of the individual or his or her spouse (Deutsches Zentrum für Altersfragen, 2016b). Given that there are different approaches and concepts regarding social stratification and division into social status groups, it could be argued that there are better or more differentiated approaches than those used in the German Ageing Survey (American Psychological Association, 2019).

\section{Conclusion}

In spite of the above limitations of this study, the findings emphasise the importance of considering both individuals' socio-economic resources and their health behaviours and age-specific attitudes in order to design (preventive) health policy programmes in older age. In addition, the study has explored the inter-relationships between individual resources, health behaviour and age-specific attitudes in a more differentiated manner than true of previous research. The findings reveal that individual resources as well as health behaviour and age-specific attitudes are independent determinants of sports participation in older age, as only slight interrelationships exist. Given the existence of social inequalities regarding health and health behaviour, policies that focus on both socio-economic conditions (which enable older people to participate in sport) and age-specific attitudes (especially for those belonging to the lowest social status group) are required.

\section{Notes}

1 In 2017, 21.4 per cent of the German population was 65 years or older. This indicates an increase of 36.6 per cent over the last 20 years (Destatis - Statistisches Bundesamt, 2018).

2 Regarding the measurement of educational attainment, institutionalised cultural capital is empirically covered here.

3 Nevertheless, it should be noted that some older people may prefer solitude and therefore do not perceive a reduction in social contacts problematic.

4 Therefore, health, wellbeing and fitness are the dominant motives for sports participation in older age (Lehnert et al., 2012; Jenkin et al., 2017).

5 The classification is based on the International Standard Classification of Education scale (Deutsches Zentrum für Altersfragen, 2016b).

6 This states whether the equivalence income is below the threshold of relative income poverty ( $60 \%$ of the median of the equivalence income of the total population) (Deutsches Zentrum für Altersfragen, 2016b). 7 Since our sub-sample contains only retired older adults, this variable categorises older adults into three groups according to their latest job position and the actual or latest job position of their spouse.

8 Response categories: 1 = 'strongly agree' to 4 = 'strongly disagree'.

9 Response categories: 1 = 'strongly disagree' to 4 = 'strongly agree'.

10 Response categories: 1 = 'strongly disagree' to 4 = 'strongly agree'.

11 Details of statistical methods, such as $\mathrm{R}$ code, can be provided by the corresponding author.

12 This refers to behavioural change theories such as the 'health belief model', 'transtheoretical model of behaviour change' and the 'health action process approach'.

Financial support. The authors declare no funding to report.

Conflict of interest. The authors declare no conflicts of interest.

Ethical standards. No statement of ethical approval is required. 


\section{References}

American Psychological Association (2019) Measuring Socioeconomic Status and Subjective Social Status. Available at https://www.apa.org/pi/ses/resources/class/measuring-status.aspx.

Arnautovska U, O'Callaghan F and Hamilton K (2018) Behaviour change techniques to facilitate physical activity in older adults: what and how. Ageing \& Society 38, 2590-2616.

Beck U (1994) Jenseits von Stand und Klasse? [Beyond status and class?]. In Beck U and Beck-Gernsheim E (eds), Riskante Freiheiten. Individualisierung in modernen Gesellschaften [Risky Freedoms. Individualization in Modern Societies]. Berlin: Suhrkamp Verlag, pp. 43-60.

Bourdieu P (1984) Distinction: A Social Critique of the Judgement of Taste. London: Routledge.

Bourdieu P (1986) The forms of capital. In Richardson J (ed.), Handbook of Theory and Research for the Sociology of Education. Westport, CT: Greenwood, pp. 241-258.

Bourdieu P (1978) Sport and social class. Information (International Social Science Council) 17, 819-840.

Breuer C, Hallmann K, Wicker P and Feiler S (2010) Socio-economic patterns of sport demand and ageing. European Review of Aging and Physical Activity 7, 61-70.

Cheng S-J, Yu H-K, Chen Y-C, Chen C-Y, Lien W-C, Yang P-Y and Hu G-C (2013) Physical activity and risk of cardiovascular disease among older adults. International Journal of Gerontology 7, 133-136.

Coleman JS (1988) Social capital in the creation of human capital. American Journal of Sociology 94, 95-120.

Conde-Sala JL, Portellano-Ortiz C, Calvó-Perxas L and Garre-Olmo J (2017) Quality of life in people aged $65+$ in Europe: associated factors and models of social welfare - analysis of data from the SHARE project (Wave 5). Quality of Life Research 26, 1059-1070.

Coxe S, West SG and Aiken LS (2009) The analysis of count data: a gentle introduction to Poisson regression and its alternatives. Journal of Personality Assessment 91, 121-136.

Daskalopoulou C, Stubbs B, Kralji C, Koukounari A, Prince M and Prina AM (2017) Physical activity and healthy ageing: a systematic review and meta-analysis of longitudinal cohort studies. Ageing Research Reviews 38, 6-17.

Del Castillo JM, Navarro JMJ-B, Sanz JLG, Rodriguez MM, Izquierdo AC and Pines DDH (2010) Being physically active in old age: relationships with being active earlier in life, social status and agents of socialisation. Ageing \& Society 30, 1097-1113.

Destatis - Statistisches Bundesamt (2017) Bevölkerung und Erwerbstätigkeit. Erwerbsbeteiligung der Bevölkerung-Ergebnisse des Mikrozensus zum Arbeitsmarkt [Population and Employment. Labour Participation of the Population - Results of the Microcensus on the Labour Market], Fachserie 1, Reihe 4.1. Wiesbaden, Germany: Destatis - Statistisches Bundesamt.

Destatis - Statistisches Bundesamt (2018) Rund jede fünfte Person ist 65 Jahre und älter [About Every Fifth Person is 65 Years and Older]. Available at https://www.destatis.de/DE/PresseService/Presse/ Pressemitteilungen/2018/09/PD18_370_12411.html.

Deutsches Zentrum für Altersfragen (2015) Deutscher Alterssurvey (DEAS): Instrumente der DEAS-Erhebung 2014. Berlin: Deutsches Zentrum für Altersfragen, Forschungsdatenzentrum.

Deutsches Zentrum für Altersfragen (2016a) Deutscher Alterssurvey (DEAS): Codebuch des DEAS 2014, Version 1.0. Berlin: Deutsches Zentrum für Altersfragen, Forschungsdatenzentrum.

Deutsches Zentrum für Altersfragen (2016b) Deutscher Alterssurvey (DEAS): Kurzbeschreibung des Datensatzes SUF DEAS2014, Version 1.0. Berlin: Deutsches Zentrum für Altersfragen, Forschungsdatenzentrum.

Ding D, Lawson KD, Kolbe-Alexander TL, Finkelstein EA, Katzmarzyk PT, van Mechelen W and Pratt M (2016) The economic burden of physical inactivity: a global analysis of major noncommunicable diseases. Lancet 388, 1311-1324.

Dionigi RA and Gard M (2018) Sport for all ages? Weighing the evidence. In Dionigi RA and Gard M (eds), Sport and Physical Activity Across the Lifespan-Critical Perspectives. London: Palgrave Macmillan, pp. 1-20.

Eime RM, Charity MJ, Harvey JT and Payne WR (2015) Participation in sport and physical activity: associations with socio-economic status and geographical remoteness. BMC Public Health 15, 434.

Emerson KG and Gay J (2017) Physical activity and cardiovascular disease among older adults: the case of race and ethnicity. Journal of Aging \& Physical Activity 25, 505-509.

Eurostat (2018) Statistics on Sport Participation. Available at https://ec.europa.eu/eurostat/statisticsexplained/index.php/Statistics_on_sport_participation. 
Gayman AM, Fraser-Thomas J, Dionigi RA, Horton S and Baker J (2017) Is sport good for older adults? A systematic review of psychosocial outcomes of older adults' sport participation. International Review of Sport and Exercise Psychology 10, 164-185.

Granovetter MS (1973) The strength of weak ties. American Journal of Sociology 78, 1360-1380.

Griffin M (2017) Embodied learning and new physical activity in mid- and later life. Qualitative Research in Sport, Exercise and Health 9, 554-567.

Grossman M (1972) On the concept of health capital and the demand for health. Journal of Political Economy 80, 223-255.

Gunasekara FI, Carter K and Blakely T (2011) Change in income and change in self-rated health: systematic review of studies using repeated measures to control for confounding bias. Social Science \& Medicine 72, 193-201.

Heinemann K (1995) Einführung in die Ökonomie des Sports: Ein Handbuch [Introduction to the Economics of Sports: A Handbook]. Schorndorf, Germany: Hofmann-Verlag.

Hoebel J, Finger JD, Kuntz B, Kroll LE, Manz K, Lange C and Lampert T (2017) Changing educational inequalities in sporting inactivity among adults in Germany: a trend study from 2003 to 2012. BMC Public Health 17, 547.

Idler EL and Benyamini Y (1997) Self-rated health and mortality: a review of twenty-seven community studies. Journal of Health and Social Behavior 38, 21-37.

Jeckel S and Sudeck G (2018) Sport activities in daily routine. Situational associations between individual goals, activity characteristics, and affective well-being. German Journal of Exercise and Sport Research 48, 26-39.

Jenkin CR, Eime RM, Wesrerbeek H, O'Sullivan G and van Uffelen GZ (2017) Sport and ageing: a systematic review of the determinants and trends of participation in sport for older adults. BMC Public Health 17, 976.

Kabacoff RI (2015) R in Action. Data Analysis and Graphics with R, 2nd Edn. Shelter Island, NY: Manning Publications.

Kohli M, Künemund H, Motel A and Szydlik M (2000) Soziale Ungleichheit [Social inequality]. In Kohli M and Künemund H (eds), Die zweite Lebenshälfte - Gesellschaftliche Lage und Partizipation im Spiegel des Alters-Survey [The Second Half of Life - Social Situation and Participation in the Mirror of the Age Survey]. Opladen: Leske + Budrich, pp. 318-336.

Lehnert K, Sudeck G and Conzelmann A (2012) Subjective well-being and exercise in the second half of life: a critical review of theoretical approaches. European Review of Aging and Physical Activity 9, $87-102$.

Lenski G (1966) Power and Privilege: A Theory of Social Stratification. New York, NY: McGraw-Hill.

Lin N (2001) Social Capital. A Theory of Social Structure and Action. Cambridge: Cambridge University Press.

Loprinzi PD, Frith E, Edwards MK, Sng E and Ashpole N (2018) The effects of exercise on memory function among young to middle-aged adults: systematic review and recommendations for future research. American Journal of Health Promotion 32, 691-704.

Marques A, Peralta M, Sarmento H, Martins J and Valeiro MG (2018) Associations between vigorous physical activity and chronic diseases in older adults: a study in 13 European countries. European Journal of Public Health 28, 950-955.

Martin M and Kliegel M (2010) Psychologische Grundlagen der Gerontopsychologie, 3., überarbeitet und erweiterte Auflage [Psychological Foundations of Gerontopsychology, 3rd, Revised and Expanded Edn]. Stuttgart, Germany: W. Kohlhammer.

Mayer K-U and Wagner M (1999) Lebenslagen und soziale Ungleichheit im hohen Alter [Life situations and social inequality in old age]. In Mayer K-U and Baltes PB (eds), Die Berliner Altersstudie [The Berlin Age Study]. Berlin: Akademie Verlag, pp. 251-275.

Molinari V, Schmid J, Sudeck G and Conzelmann A (2015) Wirkung sportlicher Aktivität auf das aktuelle Befinden im höheren Erwachsenenalter - Verlaufsanalysen in Sportprogrammen [Effect of physical activity on the current state of health in adulthood - follow-up analyses in sports programs]. Sportwissenschaft 45, 138-148.

Moschny A, Platen P, Klaaßen-Mielke R, Trampisch U and Hinrichs T (2011) Barriers to physical activity in older adults in Germany: a cross-sectional study. International Journal of Behavioral Nutrition and Physical Activity 8, 121. 
Pampel FC, Krueger PM and Denney JT (2010) Socioeconomic disparities in health behavior. Annual Review of Sociology 36, 349-370.

Phoenix C and Bell SL (2019) Beyond 'move more': feeling the rhythms of physical activity in mid and later-life. Social Science \& Medicine 231, 47-54.

Phoenix C and Orr N (2014) Pleasure: a forgotten dimension of physical activity in older age. Social Science \& Medicine 115, 94-102.

Robert Koch-Institut (ed.) (2009) Gesundheit und Krankheit im Alter. Beiträge zur Gesundheitsberichterstattung des Bundes [Health and Illness in Old Age. Contributions to Federal Health Reporting]. Berlin: RKI.

Robert Koch-Institut (ed.) (2015) Gesundheit in Deutschland. Gesundheitsberichterstattung des Bundes (Gemeinsam getragen von RKI und Destatis) [Health in Germany. Health Coverage of the Federal Government (Jointly Supported by RKI and Destatis)]. Berlin: RKI.

Shaw BA, McGeever K, Vasquez E, Agahi N and Fors S (2014) Socioeconomic inequalities in health after age 50: are health risk behaviors to blame? Social Science \& Medicine 101, 52-60.

Studer F, Schlesinger T and Engel C (2011) Socio-economic and cultural determinants of sports participation in Switzerland from 2000 to 2008. European Journal for Sport and Society 8, 147-166.

Szybalska A, Broczek K, Puzianowska-Kuznicka M, Slusarczyk P, Chudek J, Skalska A and Mossakowska M (2018) Self-rated health and its association with all-cause mortality of older adults in Poland: the PolSenior project. Archives of Gerontology and Geriatrics 79, 13-20.

Thiel A, Huy C and Gomolinsky U (2008) Alterssport in Baden-Württemberg - Präferenzen, Motive und Settings für die Sportaktivität in der Generation $50+$. [Exercise and sports among older adults in Baden-Wuerttemberg, Germany-preferences, motives and settings for sporting activity in the 50-plus generation]. Deutsche Zeitschrift für Sportmedizin 59, 163-167.

Tulle E and Dorrer N (2012) Back from the brink: ageing, exercise and health in a small gym. Ageing e Society 32, 1106-1127.

Wanner M, Tarnutzer S, Martin BW, Braun J, Rohrmann S, Bopp M and Faeh D (2014) Impact of different domains of physical activity on cause-specific mortality: a longitudinal study. Preventive Medicine 62, 89-95.

Wicker P and Frick B (2015) The relationship between intensity and duration of physical activity and subjective well-being. European Journal of Public Health 25, 868-872.

Willey JZ, Moon YP, Sherzai A, Cheung YK, Sacco RL and Elkind MSV (2015) Leisure-time physical activity and mortality in a multiethnic prospective cohort study: the Northern Manhattan Study. Annals of Epidemiology 25, 475-479.

Wollesen B and Voelcker-Rehage C (2013) Training effects on motor-cognitive dual-task performance in older adults. European Review of Aging and Physical Activity 11, 5-24.

World Health Organization (WHO) (2010) Global Recommendations on Physical Activity for Health. Geneva: WHO.

Wurm S, Berner F and Tesch-Römer C (2013) Altersbilder im Wandel [Age Pictures in Change]. Bundeszentrale für politische Bildung. Available at http://www.bpb.de/apuz/153117/altersbilder-im-wandel?p=all.

Cite this article: Faß E, Schlesinger T (2021). The role of individual resources, health behaviour and age perception as determinants of sports participation in older age. Ageing \& Society 41, 746-772. https:// doi.org/10.1017/S0144686X19001260 